\title{
RELIABILITY INDEX FOR IRIS CODE MATCHING IN IRIS RECOGNITION SYSTEMS
}

\author{
B. H. Shekar ${ }^{\mathrm{a}, *}$, Sharada S Bhat ${ }^{\mathrm{b}}$, Archil Maysuradze \\ ${ }^{a}$ Mangalore University, Mangalore, Karnataka India - bhshekar@gmail.com \\ ${ }^{\mathrm{b}}$ Government First Grade College, Ankola, Karnataka, India - sharadasbhat@ gmail.com \\ ${ }^{\mathrm{c}}$ Lomonosov Moscow State University, Moscow, Russia - artchil@mail.ru
}

Commission II, WG II/10

KEY WORDS: Iris code matching, Reliability Index, Iris recognition

\begin{abstract}
:
Iris code matching is an important stage of iris biometric systems which compares the input iris code with stored patterns of enrolled iris codes and classifies the code into one of classes so that, the claim is accepted or rejected. Several classifier based approaches are proposed by the researchers to improve the recognition accuracy. In this paper, we discuss the factors affecting an iris classifier's performance and we propose a reliability index for iris matching techniques to quantitatively measure the extent of system reliability, based on false acceptance rate and false rejection rates using Monte Carlo Simulation. Experiments are carried out on benchmark databases such as, IITD, MMU v-2, CASIA v-4 Distance and UBIRIS v.2.
\end{abstract}

\section{INTRODUCTION}

Iris code matching and classifying is a crucial stage in any iris recognition system. During this stage, the query iris code is compared with an enrolled iris code to generate a match score. Match score is a similarity or a dissimilarity measure which is computed using a distance function. If it is authentication (verification) system, the query iris code is compared with the iris patterns of the claimer so that, the database of enrolled iris codes to be searched is smaller in size and hence, the number of match scores generated are less. Where as, in case of identification systems, query iris code is compared with all the enrolled iris patterns so that the database size is quite larger and hence, large number of match scores are generated. However, in both the systems, a classifier (iris code matcher) has to be adopted at this stage. In authentication systems the iris matcher has to accept or reject the claim (binary classification) and in identification systems, the classifier has to classify the query iris code into one of the $N+1$ classes where $N$ is the number of subjects enrolled in the database (multi-class classifier). In password-based recognition systems a perfect match between the two password strings is necessary. Where as, in biometric recognition systems, identity of a person is determined based on a close match between query and enrolled iris codes. Closeness of the match score is decided by a threshold value. (Jain et al., 2011).

Several approaches have been proposed in the literature for iris code matching (Bowyer et al., 2008, Bowyer and Burge, 2016). The well known Daugman's approach (Daugman, 1993) uses Hamming distance with exclusive OR operator (XOR operator) to generate a dissimilarity score between the two iris codes and statistical decision theory for classification. Two iris codes with extracted features are not all statistically independent of each other. However, given the Hamming distance distributions for a large number of genuine match scores (scores between the two irises of the same subject-mate irises) and impostor match scores (scores between the two irises of different subjects-nonmate irises), the distributions can be fit with a binomial curve to find the effec-

\footnotetext{
${ }^{*}$ Corresponding author
}

tive number of degrees of freedom which calculates a confidence limit for a match of two iris codes. To measure the goodness-ofmatch between the feature vectors of query iris and enrolled irises Wildes et.al (Wildes, 1997) have used normalised correlation of the feature vectors and have utilised Fisher's linear discriminant function for decision making. Le Ma et.al. (Ma et al., 2004) have devised a matching scheme on the expanded feature vectors based on XOR operator. For comparing the two iris codes, a nearest neighbor approach is used by Monro et.al. (Monro et al., 2007) and to compute the distance they have proposed product-of-sum of Hamming distance of individual sub features extracted from each iris. These early methods perform well with the constrained imaging systems. However, recent researchers are working with the noisy iris images taken at-a-distance and in the relaxed imaging scenario wherein, the images suffer from occlusion, bad illuminations and reflections (Nguyen et al., 2017) and the research is referred to as unconstrained iris recognition.

In order to undermine the effect of noise in unconstrained iris recognition, the localised iris region is divided into $N$ subregions (called as patches) using $m$ tracks and $n$ sectors. Proenca and Allexandre (Proenca and Alexandre, 2007) have extracted the features of individual patch, computed the match score between the corresponding patches and then proposed a classification rule to combine the scores. Hollingsworth et.al. (Hollingsworth et al., 2011) have proposed a fragile bit distance, the match score obtained by which, is then combined with the hamming distance. Wang et.al. (Wang et al., 2012) have adopted Adaboost learning to determine the iris patches with most discriminative features. Pillai et.al. (Pillai et al., 2011) have divided the unwrapped iris image into four sectors, extracted the features from each sector and used a sparse based recognition algorithm for classification. Different distance measures and classifiers are existing in the literature are Euclidean distance (Proença and Briceño, 2013, Tan and Kumar, 2013), $\chi^{2}$ distance, (Bharadwaj et al., 2010), normalised cosine distance, (Juefei-Xu and Savvides, 2012), supervised Mahalanobis distance(Nie et al., 2014) and weighted mean sum.

The key question is : Is there any reason to prefer one classifier 
over the other? As stated by No-free-lunch theorem (Wolpert and Macready, 1997), any learning algorithm which performs better for a particular class of pattern recognition (PR) problem is not superior in general for all PR problems. The ugly-duckling theorem (Kamishima et al., 2012) states that there is no feature (no pattern representation) that will yield better classification performance, unless we make some assumptions about the data. In (Kryszczuk et al., 2005), authors have presented a framework to predicate and correct the classification errors on modality reliability measures in multi-modal biometric system, with the case study of face and speech recognition.

Though, the existing performance measures of biometric recognition systems measure the efficiency of the systems, they don't answer the question, how to select a better classifier for an iris recognition system. In this paper, we propose a reliability index $(R I)$ to measure the performance of iris classifiers. We define $R I$ based on false acceptance rate (FAR) and false reject rate (FRR) of the classifier's output. We propose Monte Carlo simulation to compute $R I$ thus defined. We also discuss the various factors that affect the reliability of iris code matching techniques.

Rest of the paper is organised as follows. Section 2 briefs the existing methods of measurements of performance of iris recognition systems. In Section 3 we explain the proposed reliability index and how to compute this using Monte Carlo simulation method. The experimental illustration of the proposed $R I$ is discussed in Section 4 and Section 5 concludes this paper.

\section{EXISTING MEASURES}

Based on Daugman's statistical decision theory (Daugman, 1993), in case of iris authentication systems, failure of iris classifiers is measured by the two error rates, false acceptance rate (FAR), accepting a non-mate iris as mate iris and false reject rate (FRR), rejecting a mate iris as non-mate iris. The goal of any iris classifier is to minimise these two error rates. Let $\omega_{g}$ and $\omega_{i}$ denote genuine and impostor classes respectively and $P\left(x \mid \omega_{g}\right)$ and $P\left(x \mid \omega_{i}\right)$ be their probability distributions where $x$ is a variable representing an arbitrary dissimilarity score. Figure 1 presents an example of $P\left(x \mid \omega_{g}\right)$ and $P\left(x \mid \omega_{i}\right)$ computed with respect to MMU v.2 iris dataset using the methods discussed in (Masek et al., 2003).

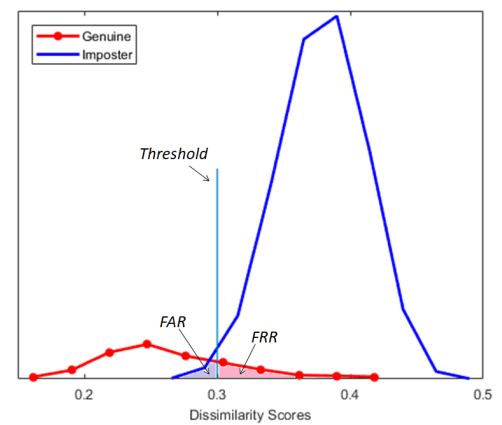

Figure 1. Probability distributions of genuine and impostor scores $P\left(x \mid \omega_{g}\right)$ and $P\left(x \mid \omega_{i}\right)$.

FAR is the probability of an impostor score $x$ to be accepted by the classifier, where as, FRR is the probability of a genuine score to be rejected. Since these probabilities change with respect to the system threshold $\tau$, they are defined as functions of $\tau$, as follows (Jain et al., 2011).

$$
\begin{aligned}
& F A R(\tau)=P\left(x \leq \tau \mid \omega_{i}\right)=\int_{-\infty}^{\tau} P\left(x \mid \omega_{i}\right) d x \\
& F R R(\tau)=P\left(x>\tau \mid \omega_{g}\right)=\int_{\tau}^{\infty} P\left(x \mid \omega_{g}\right) d x
\end{aligned}
$$

Further, by computing FAR and FRR at various threshold values two smooth curves, detection error tradeoff (DET) and receiver operating characteristic (ROC), can be obtained which are the graphical tools to measure the performance of iris authentication systems. DET curve is obtained by plotting FRR against FAR at different threshold values on a normal deviate scale and ROC curve plots true acceptance rate (1-FRR) against FAR using linear logarithmic scale. Iris authentication systems can also be evaluated by single valued measures like, equal error rate (EER) which is the point on DET curve where FAR equals FRR and $d$-prime value which measures the separation between the probability distributions $P\left(x \mid \omega_{g}\right)$ and $P\left(x \mid \omega_{i}\right)$ and is defined as follows.

$$
d-\text { prime }=\frac{\left|\mu_{i}-\mu_{g}\right|}{\sqrt{\frac{1}{2}\left(\sigma_{i}^{2}+\sigma_{g}^{2}\right)}}
$$

where, $\mu_{i}$ and $\sigma_{i}$ are the mean and standard deviation of impostor distribution and $\mu_{g}$ and $\sigma_{g}$ are those of genuine distribution.

Researchers have been using these measuring tools to prove the efficiency of their algorithms with respect to the best performance on a dataset. However, these tools do not provide any clue about how reliable an algorithm is, with respect to a random scenario. We propose an index which measures the reliability of a matching algorithm when the algorithm is applied on different datasets. Similar to $d$ - prime or EER, proposed $R I$ is also single valued measure which can tell whether a classifier is reliable against the other classifiers in a given scenario.

\section{PROPOSED RELIABILITY INDEX}

Reliability of a system means the ability of the system to fulfill what is required of it. Quantitatively it is measured as the probability that a system operates successfully (Crowder, 2017). Statistical meaning of simple reliability index for a system with random variables which are described by their mean values, variances and covariances (Ditlevsen and Madsen, 1996) is explained in the next section.

\subsection{Statistical meaning of RI}

Assume that the input variables $\left\{x_{1}, x_{2}, \ldots, x_{n}\right\}$ with some uncertainties are modeled as random variables $\left\{X_{1}, X_{2}, \ldots, X_{n}\right\}$ with the given mean values,

$$
E\left[X_{1}\right], E\left[x_{2}\right], \ldots, E\left[X_{n}\right]
$$

and covariances are defined by,

$$
\operatorname{Cov}\left[X_{i}, X_{j}\right]=E\left[X_{i}, X_{j}\right]-E\left[X_{i}\right] E\left[X_{j}\right]
$$

Let $R$ be a new random variable given by,

$$
R=a_{1} X_{1}+a_{2} X_{2}+\ldots+a_{n} X_{n}+b=a^{T} X+b
$$

where $a=\left[a_{1}, a_{2}, \ldots, a_{n}\right], X=\left(X_{1}, X_{2}, \ldots, X_{n}\right)$ and $a_{1}, a_{2}, \ldots, a_{n}$ and $b$ are given constants. The mean and variance of the variable 


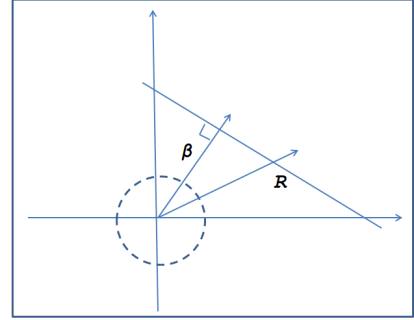

Figure 2. Geometric interpretation of simple reliability index.

$R$ are defined as,

$$
\begin{gathered}
E[R]=a^{T} E[X]+b \\
\operatorname{Var}[R]=a^{T} \operatorname{Cov}\left[X, X^{T}\right] a
\end{gathered}
$$

Then, simple reliability index is denoted by $\beta$ and is defined as the ratio of mean and standard deviation, where, standard deviation is given by the square root of variance.

$$
\beta=\frac{E[R]}{\sqrt{\operatorname{Var}[R]}}=\frac{a^{T} E[X]+b}{\sqrt{a^{T} \operatorname{Cov}\left[X, X^{T}\right] a}}
$$

If the random variable $R$ defined in equation (6) is normalised with zero mean and unit standard deviation then, geometrically simple reliability index is the perpendicular distance from the origin to the hyperplane represented by normalised $R$ (see Figure 2).

\subsection{Reliability index for iris classifiers}

Apart from the statistical definition, in general, $R I$ of any system is defined based on the probability of the failure of the system.

$$
R I=1-\text { Probability (failure) }
$$

We define $R I$ in terms of probability of failure using equation (10). Since our definition is based on FAR and FRR, $R I$ is also a function of system threshold $\tau$.

Definition 3.1. Let $\omega_{\tau}^{F A R}$ and $\omega_{\tau}^{F R R}$ represent the classes of dissimilarity scores falling under false acceptance and false reject regions respectively for a particular value of $\tau$, so that, both are subsets of $\omega_{g} \cap \omega_{i}$, then, the probability of failure is given by,

$$
P\left(x \mid \omega_{\tau}^{F A R} \cup \omega_{\tau}^{F R R}\right)
$$

Hence, the reliability index $R I$ is given by,

$$
R I(\tau)=1-P\left(x \mid \omega_{\tau}^{F A R} \cup \omega_{\tau}^{F R R}\right)
$$

Since, $\omega_{\tau}^{F A R}$ and $\omega_{\tau}^{F R R}$ are disjoint classes, it can be further simplified as,

$$
\begin{aligned}
R I(\tau) & =1-\left(P\left(x \mid \omega_{\tau}^{F A R}\right)+P\left(x \mid \omega_{\tau}^{F R R}\right)\right) \\
& =1-\left(P\left(x \leq \tau \mid \omega_{i}\right)+P\left(x>\tau \mid \omega_{g}\right)\right)
\end{aligned}
$$

Using equations (1) and (2) we can write $R I$ as,

$$
R I(\tau)=1-(F A R(\tau)+F R R(\tau))
$$

RI given in equation(14) is trivial and straight forward. However, the reliability of an iris classifier is affected by several other factors also. Intermingling all the effects together, we propose the following expression for $R I$ using a normalising factor $\eta$.

$$
R I(\tau)=1-\frac{1}{\eta}(F A R(\tau)+F R R(\tau))
$$

In the following section we discuss factors that affect a classifier's performance in iris authentication systems and how the expression $\eta$ can be computed.

\subsection{Factors affecting the iris classifier's performance}

The most influential factor that affects an iris classifier's performance is iris training dataset. Publicly available near infrared (NIR) and visual wavelength(VW) iris datasets possess variety of degraded images for training the classifiers. These degraded images, unless handled properly, reduce the recognition accuracy, thereby making the system less reliable. In a recent paper (Kabra et al., 2015) authors have proposed a distance measure which helps to tune the most influential training dataset that causes classifier's errors. Size of the dataset and the training to test ratio are the other factors that affect the performance. In the applications like, bank teller machines, search for the best match is against a smaller and specific set of iris templates and such authentication systems are expected to possess zero percentage of false acceptance rate (FAR) with highest possible reliability index. i.e. such applications require the systems with $R I=1$.

In (Liu et al., 2018), authors have proposed inner reliability of a classifier, represented by a matrix $R_{c \times c}$ ( $c$ being the number of classes in the data set), to characterize the conditional probability of the object belonging to class number $i(i=1, \ldots, c)$ when it is classified into class number $j(j=1,2, \ldots, c)$ by the given classifier. Authors have proposed a method to evaluate the reliability matrix using training information and they claim that this matrix provides much more refined reliability knowledge than the training accuracy value.

Thus, considering the effect of training datasets, we take $\eta$ to be proportional to the total size of the classes $\omega_{i}$ and $\omega_{g}$.

$$
\eta \propto\left|\omega_{i} \cup \omega_{g}\right|
$$

In addition, $R I$ is also affected by the segmentation errors and the filtering errors of the feature extraction algorithms and hence, $\eta$ also depends on the percentages of segmentation and filtering error.

\subsection{Application of Monte Carlo Simulation}

To compute the reliability index, several statistical methods are discussed in (Crowder, 2017) and (Ditlevsen and Madsen, 1996). Terry and Mae (Koo and Li, 2016) have used intra-class correlation coefficient for reliability analysis in clinical research. Monte Carlo simulation (MCS) has been a powerful tool for reliability assessment of distribution systems in various fields (Liang and Goel, 1997). MCS is based on the probability concept that, while behavior of average samples of random variables is mathematically established, these behavioral tendency is stabilized as the sample size increases largely. The necessary condition to apply MCS is the randomness of the input variables which is well satisfied in case of probability distribution of iris dissimilarity scores.

To explain the application of MCS for evaluation of RI in case of iris classifiers, an arbitrary dissimilarity score $x$ may fall in any area of the distributions $P\left(\omega_{g}\right)$ and $P\left(\omega_{i}\right)$ (Refer Figure 1). When experimented with a small sample of dissimilarity scores, 


\begin{tabular}{|l|c|c|c|c|}
\hline Method & IITD & $\begin{array}{c}\text { MMU } \\
\text {-2 }\end{array}$ & $\begin{array}{c}\text { CASIA } \\
\text { v-4 Dist }\end{array}$ & $\begin{array}{c}\text { UBIRIS } \\
\text { v.2 }\end{array}$ \\
\hline \hline DCT & 0.9560 & 0.9355 & 0.9470 & 0.9332 \\
LOG-Gabor & 0.9638 & 0.9500 & 0.9543 & 0.9500 \\
Riesz & 0.9773 & 0.9673 & 0.9643 & 0.9401 \\
TSE & 0.9793 & 0.9681 & 0.9649 & 0.9365 \\
\hline
\end{tabular}

Table 1. $R I$ obtained by the experiments conducted using different filters with respect to the four databases. Distance measure is the Hamming distance in all the cases.

the number of scores in the area of $P\left(\omega_{\tau}^{F A R}\right)$ and $P\left(\omega_{\tau}^{F R R}\right)$ are counted for each $\tau$ and $R I$ is computed using equation (15). MCS is then carried out with the increased size of sample space to get the final average value of RI. Size of the class of iris scores is sufficiently large, having more than a lakh of impostor scores.

\section{EMPIRICAL ILLUSTRATION}

In this section we empirically demonstrate that using the proposed reliability index one can choose a suitable matching algorithm for the given dataset. We have conducted the experiments on publicly available bench mark databases, IIT Delhi (Indian Institute of Technology, Delhi, India, Iris Database, n.d.), MMU v-2 (Malaysia Multimedia University Iris Database, n.d.), CASIA v-4 Distance (Institute of Automation, Chinese Academy of Sciences. CASIA Iris Database, n.d.) and UBIRIS v.2 (Proenca et al., 2010). The first three datasets consists of near infra red (NIR) images where as, UBIRIS databse has visual wave length(VW) images.

For iris segmentation we have adopted the method proposed by (Proenca, 2010). In order to extract the iris features, we have adopted the filters, DCT (Monro et al., 2007), Log-Gabor (Masek et al., 2003), Riesz (Shekar and Bhat, 2015) and TSE (Shekar and Bhat, 2016). Daugman's rubber sheet model and phase encoding techniques are used for unwrapping the iris and encoding the features respectively.

During the first set of experiments the $R I$ is computed on the four datasets using the distance measure as Hamming distance, with respect to different feature extraction filters. The normalising factor $\eta$ is separately calculated for all the datasets and for all the filters. The results of these experiments are presented in Table 1. The number of system threshold $\tau$ is varied from 20 to 2500 and for the smallest of the datasets, MMU v-2, a total of 487080 dissimilarity scores are generated to carry out the simulation. The results displayed in Table 1 depict that some of the feature extraction techniques are more reliable with respect to particular database. That means $R I$ can also be applied to test the various iris feature extraction techniques. It has also been observed that with respect to the filters used, NIR imaging is more reliable compared to VW scenario.

Second set of experiments are conducted by using the same filter but using different distance measures. Experiments are conducted using Riesz filter on different datasets. During these experiments the $\eta$ is based only on the size of dataset, the factors based on the segmentation errors and filtering errors are kept constant. $R I$ of Hamming distance (HD) is compared with that of weighted mean Hamming distance (WMHD). Segmented iris is divided into $N$ patches and each patch is assigned with some weight and the difference between the two iris codes is computed using WMHD.

\begin{tabular}{|l|c|c|}
\hline Datasets & HD & WMHD \\
\hline \hline IITD & 0.9773 & 0.9800 \\
MMU v-2 & 0.9673 & 0.9770 \\
CASIA v-4 Distance & 0.9634 & 0.9765 \\
UBIRIS v.2 & 0.9401 & 0.9760 \\
\hline
\end{tabular}

Table 2. $R I$ obtained by using Riesz filter with two different distance measures HD and WMHD, on all the databases.

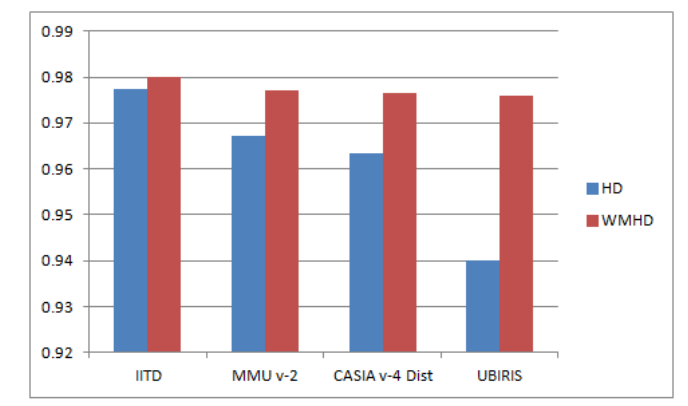

Figure 3. $R I$ showing that, with respect to all databases, WHMD is more reliable than normal Hamming distance.

Reader can refer (Shekar and Bhat, 2017) for detailed explanation. Table 2 and Figure 3 present the results of second set of experiments. Figure 3 shows that with respect to all databases WHMD is more reliable than normal Hamming distance.

During the third set of experiments, we have computed the dprime (using the equation (3)) and $R I$ values on the same dataset (IITD) with respect to different filters. In these experiments $\eta$ is based only on the filtering errors. Results presented in Table 3 illustrate that both $R I$ and $d$-prime are positively correlated. Though, $d$-prime is one of the popular performance measures of biometric systems, proposed $R I$ checks the extent to which the algorithm is reliable.

\section{CONCLUSION}

We have proposed a quantitative measure for reliability $(R I)$ for iris code matching techniques. Proposed $R I$ is tested by conducting rigorous experiments on different data sets using different feature extraction methodologies and matching techniques. We also have discussed the various factors that affect the performance of an iris code matcher. It has been observed that a technique which is more reliable on one particular system may not be the superior in context of another system.

Proposed $R I$ is based on the two error rates FAR and FRR which are based on the authentication system errors. When we consider the errors in identification systems, we get two more error

\begin{tabular}{|l|c|c|c|c|}
\hline \multirow{2}{*}{ Method } & \multicolumn{2}{|c|}{ HD } & \multicolumn{2}{c|}{ WMHD } \\
\cline { 2 - 5 } & $R I$ & $d-$ prime & $R I$ & $d-$ prime \\
\hline \hline DCT & 0.9500 & 2.7604 & 0.9560 & 3.2100 \\
\hline Log-Gabor & 0.9638 & 5.4817 & 0.9679 & 5.5033 \\
\hline Riesz & 0.9773 & 5.6306 & 0.9800 & 5.6311 \\
\hline TSE & 0.9793 & 5.8728 & 0.9816 & 5.8796 \\
\hline
\end{tabular}

Table 3. $R I$ and $d$-prime values obtained by different filters and two distance measures HD and WMHD, on IITD database. 
rates, false positive identification rate (FPIR) and false negative identification rate (FNIR). By making minor modifications in the expression given in equation (15), $R I$ can also be computed for iris identification systems.

\section{ACKNOWLEDGEMENT}

This work is supported jointly by the Department of Science \& Technology, Govt. of India and Russian Foundation for Basic Research, Russian Federation under the grant No. INT/RUS / RFBR /P-248.

\section{REFERENCES}

Bharadwaj, S., Bhatt, H. S., Vatsa, M. and Singh, R., 2010. Periocular biometrics: When iris recognition fails. In: BTAS, pp. 1-6.

Bowyer, K. W. and Burge, M. J., 2016. Handbook of iris recognition. Springer.

Bowyer, K. W., Hollingsworth, K. and Flynn, P. J., 2008. Image understanding for iris biometrics: A survey. Computer vision and image understanding 110(2), pp. 281-307.

Crowder, M. J., 2017. Statistical analysis of reliability data. Routledge.

Daugman, J. G., 1993. High confidence visual recognition of persons by a test of statistical independence. IEEE transactions on pattern analysis and machine intelligence 15(11), pp. 11481161.

Ditlevsen, O. and Madsen, H. O., 1996. Structural reliability methods. Vol. 178, Wiley New York.

Hollingsworth, K. P., Bowyer, K. W. and Flynn, P. J., 2011. Improved iris recognition through fusion of hamming distance and fragile bit distance. IEEE transactions on pattern analysis and machine intelligence 33(12), pp. 2465-2476.

Indian Institute of Technology, Delhi, India, Iris Database, n.d. http://www4. comp.polyu.edu.hk/csajaykr/IITD/ Database_Iris.htm/.

Institute of Automation, Chinese Academy of Sciences. CASIA Iris Database, n.d. http://biometrics.idealtest.org/.

Jain, A. K., Ross, A. A. and Nandakumar, K., 2011. Introduction to biometrics. Springer Science \& Business Media.

Juefei-Xu, F. and Savvides, M., 2012. Unconstrained periocular biometric acquisition and recognition using cots ptz camera for uncooperative and non-cooperative subjects. In: Applications of Computer Vision (WACV), 2012 IEEE Workshop on, IEEE, pp. 201-208.

Kabra, M., Robie, A. and Branson, K., 2015. Understanding classifier errors by examining influential neighbors. In: Proceedings of the IEEE conference on computer vision and pattern recognition, pp. 3917-3925.

Kamishima, T., Akaho, S., Asoh, H. and Sakuma, J., 2012. Enhancement of the neutrality in recommendation. In: Decisions@ RecSys, pp. 8-14.

Koo, T. K. and Li, M. Y., 2016. A guideline of selecting and reporting intraclass correlation coefficients for reliability research. Journal of chiropractic medicine 15(2), pp. 155-163.

Kryszczuk, K., Richiardi, J., Prodanov, P. and Drygajlo, A., 2005. Error handling in multimodal biometric systems using reliability measures. In: Signal Processing Conference, 2005 13th European, IEEE, pp. 1-4.
Liang, X. and Goel, L., 1997. Distribution system reliability evaluation using the monte carlo simulation method. Electric Power systems research 40(2), pp. 75-83.

Liu, Z., Pan, Q., Dezert, J., Han, J.-W. and He, Y., 2018. Classifier fusion with contextual reliability evaluation. IEEE transactions on cybernetics $48(5)$, pp. 1605-1618.

Ma, L., Tan, T., Wang, Y. and Zhang, D., 2004. Efficient iris recognition by characterizing key local variations. IEEE Transactions on Image processing 13(6), pp. 739-750.

Malaysia Multimedia University Iris Database, n.d. http:// pesona.mmu.edu.

Masek, L. et al., 2003. Recognition of human iris patterns for biometric identification.

Monro, D. M., Rakshit, S. and Zhang, D., 2007. Dct-based iris recognition. IEEE Transactions on Pattern Analysis and Machine Intelligence 29(4), pp. 586-595.

Nguyen, K., Fookes, C., Jillela, R., Sridharan, S. and Ross, A., 2017. Long range iris recognition: A survey. Pattern Recognition 72, pp. 123-143.

Nie, L., Kumar, A. and Zhan, S., 2014. Periocular recognition using unsupervised convolutional rbm feature learning. In: 2014 22nd International Conference on Pattern Recognition (ICPR), IEEE, pp. 399-404.

Pillai, J. K., Patel, V. M., Chellappa, R. and Ratha, N. K., 2011. Secure and robust iris recognition using random projections and sparse representations. IEEE transactions on pattern analysis and machine intelligence 33(9), pp. 1877-1893.

Proenca, H., 2010. Iris recognition: On the segmentation of degraded images acquired in the visible wavelength. IEEE Transactions on Pattern Analysis and Machine Intelligence 32(8), pp. 1502-1516.

Proenca, H. and Alexandre, L. A., 2007. Toward noncooperative iris recognition: A classification approach using multiple signatures. IEEE Transactions on Pattern Analysis and Machine Intelligence.

Proença, H. and Briceño, J. C., 2013. Periocular biometrics: constraining the elastic graph matching algorithm to biologically plausible distortions. IET Biometrics 3(4), pp. 167-175.

Proenca, H., Filipe, S., Santos, R., Oliveira, J. and Alexandre, L. A., 2010. The UBIRIS.v2: A database of visible wavelength iris images captured on-the-move and at-a-distance. IEEE Transactions on Pattern Analysis and Machine Intelligence 32(8), pp. 1529-1535.

Shekar, B. and Bhat, S. S., 2015. Steerable riesz wavelet based approach for iris recognition. In: Pattern Recognition (ACPR), 2015 3rd IAPR Asian Conference on, IEEE, pp. 431-436.

Shekar, B. and Bhat, S. S., 2016. Iris recognition using partial sum of second order taylor series expansion. In: Proceedings of the Tenth Indian Conference on Computer Vision, Graphics and Image Processing, ACM, p. 81.

Shekar, B. and Bhat, S. S., 2017. Multi-patches iris based person authentication system using particle swarm optimization and fuzzy c-means clustering. The International Archives of Photogrammetry, Remote Sensing and Spatial Information Sciences 42, pp. 243.

Tan, C.-W. and Kumar, A., 2013. Towards online iris and periocular recognition under relaxed imaging constraints. IEEE Transactions on Image Processing 22(10), pp. 3751-3765. 
Wang, Q., Zhang, X., Li, M., Dong, X., Zhou, Q. and Yin, Y., 2012. Adaboost and multi-orientation $2 d$ gabor-based noisy iris recognition. Pattern Recognition Letters 33(8), pp. 978-983.

Wildes, R. P., 1997. Iris recognition: an emerging biometric technology. Proceedings of the IEEE 85(9), pp. 1348-1363.

Wolpert, D. H. and Macready, W. G., 1997. No free lunch theorems for optimization. IEEE transactions on evolutionary computation 1(1), pp. 67-82. 